\title{
THEOREM OF LINDEBERG IN THE CALCULUS OF VARIATIONS
}

BY

MAGNUS R. HESTENES

1. Introduction. The present paper is the second of a sequence of three papers concerned with the isoperimetric problem of Bolza in parametric form. The first paper $\left(^{1}\right)$ is concerned with certain properties of the Weierstrass $E$-function that will be useful in the present paper and in the one to follow. The third paper will be concerned with a sufficiency theorem conjectured by McShane $\left({ }^{2}\right)$. In the present note is found an extension of the theorem of Lindeberg and related results. This theorem is an obvious extension to the parametric case of a similar theorem given by Reid $\left(^{3}\right)\left({ }^{4}\right)$ for the nonparametric case and is an immediate consequence of the arguments used by Reid in an expansion proof for the parametric problems which has not been published as yet. The approach to this theorem here given is different from that given by Reid. Moreover the theorem is stated so as to bring out a condition of uniformity that simplifies the applications of the theorem.

Among the many consequences of the theorem of Lindeberg and a related theorem is the equivalence of the sufficiency theorems for the problems of Mayer and Bolza, a result that does not appear to have been established completely heretofore. It is shown moreover that the sufficiency theorem for the isoperimetric problem of Bolza can be obtained from those for the problem of Bolza without isoperimetric conditions. It is also pointed out that the sufficiency theorems for parametric problems can be obtained from those for the nonparametric problems. In addition to further results of similar nature, we give in the last section a result that will be useful in the paper to follow. This result is the only part of the present paper that is explicitly used in the next paper and is based on the results given in $\$ \$ 2,3,4,5,11$ below.

2. Hypotheses and notations. The problem of Bolza as formulated in the first paper is that of finding in a class of arcs

Presented to the Society, April 27, 1946; received by the editors November 9, 1945.

(1) M. R. Hestenes, The Weierstrass E-function in the calculus of variations, Trans. Amer. Math. Soc. vol. 60 (1946) pp. 51-71.

(2) E. J. McShane, Sufficient conditions for a weak relative minimum, Trans. Amer. Math. Soc. vol. 52 (1942) p. 346.

(3) W. T. Reid, Isoperimetric problems of Bolza in nonparametric form, Duke Math. J. vol. 5 (1939) pp. 625-691.

(4) See also I. E. Perlin, Sufficient conditions in the problem of Lagrange with isoperimetric side conditions, Contributions to the calculus of variations, 1933-1937, The University of Chicago Press. 
$C: \quad a^{h}, \quad y^{i}(t) \quad\left(t^{1} \leqq t \leqq t^{2} ; h=1, \cdots, r ; i=0,1, \cdots, n\right)$ satisfying a set of conditions of the form

$$
\begin{array}{cc}
\phi^{\beta}(a, y, \dot{y})=0 & (\beta=1, \cdots, m<n), \\
y^{i}\left(t^{1}\right)=T^{i 1}(a), \quad y^{i}\left(t^{2}\right)=T^{i 2}(a), & \\
I^{\sigma}(C)=g^{\sigma}(a)+\int_{C} f^{\sigma}(a, y, \dot{y}) d t=0 & (\sigma=1, \cdots, s)
\end{array}
$$

one which minimizes the function

$$
I(C)=g(a)+\int_{C} f(a, y, \dot{y}) d t .
$$

The hypotheses, notations and terminology described in the first paper( $\left(^{(5)}\right.$. will be used throughout. Thus, an arc $C$ is denoted by

C:

$$
a, y(t)
$$$$
\left(t^{1} \leqq t \leqq t^{2}\right) ;
$$

it is admissible if it satisfies equations (2.1), (2.2), and (2.3). As before we assume that we have given an admissible arc

$C_{0}$ :

$$
a_{0}, y_{0}(t)
$$$$
\left(t^{1} \leqq t \leqq t^{2}\right)
$$

of class $C^{\prime \prime}$ that does not intersect itself and along which the matrix

$$
\left\|\phi_{p^{i}}^{\beta}\left[a_{0}, y_{0}(t), \dot{y}_{0}(t)\right]\right\|
$$

has rank $m$ on $t^{1} t^{2}$. Throughout this paper it will be assumed that there exists a set of continuous multipliers

$$
l^{0} \geqq 0, \quad l^{\sigma}, \quad m^{\beta}(a, y)
$$

such that the function

$$
F(a, y, p)=l^{0} f(a, y, p)+l^{\sigma} f^{\sigma}(a, y, p)+m^{\beta}(a, y) \phi^{\beta}(a, y, p)
$$

$E$-dominates $L(p)=|p|$ and $f^{\sigma}(a, y, p)$ near $C_{0}$ on $\mathfrak{D}$, where $\mathfrak{D}$ is the set of admissible elements $(a, y, p)$ having $\phi^{\beta}(a, y, p)=0$. In other words there exists a constant $b>0$ and a neighborhood $\mathfrak{D}_{0}$ of $C_{0}$ relative to $\mathfrak{D}$ such that the inequalities

$$
\begin{aligned}
E_{L}(p, q) & \leqq b E_{F}(a, y, p, q), \\
\left|E_{f^{\sigma}}(a, y, p, q)\right| & \leqq b E_{F}(a, y, p, q) \quad(\sigma=1, \cdots, s)
\end{aligned}
$$

hold whenever $(a, y, p)$ is in $\mathfrak{D}_{0}$ and $(a, y, q)$ is in $\mathfrak{D}$. Here and elsewhere $E_{H}$ is the Weierstrass $E$-function

$$
E_{H}(a, y, p, q)=H(a, y, q)-q^{i} H_{p^{i}}(a, y, p)
$$

(5) M. R. Hestenes, loc. cit. \$2. 
for an admissible function $H(a, y, p)$. As was seen in the preceding paper this assumption is equivalent to the assumption of nonsingularity together with the strengthened condition of Weierstrass. By Theorem 4.3 of this first paper we can suppose further that $b$ and $\mathfrak{D}_{0}$ have been chosen so that one also has

$$
\left|E_{\phi \beta}(a, y, p, q)\right| \leqq b E_{F}(a, y, p, q) \quad(\beta=1, \cdots, m)
$$

whenever $(a, y, p)$ is in $\mathfrak{D}_{0}$ and $(a, y, q)$ is in $\mathfrak{D}$.

In the following pages we shall be interested in studying the properties of the function

$$
J(C)=G(a)+\int_{C} F(a, y, \dot{y}) d t
$$

where $F(a, y, p)$ is given by $(2.6)$ and

$$
G(a)=l^{0} g(a)+l^{\sigma} g^{\sigma}(a) .
$$

It is clear that

$$
J(C)=l^{0} I(C)+l^{\sigma} I^{\sigma}(C)+\int_{C} m^{\beta}(a, y) \phi^{\beta} d t
$$

and hence that

$$
J(C)=l^{0} I(C)
$$

for every admissible arc $C$. Thus if the constant $l^{0}>0$, a study of the function $J(C)$ is equivalent to the study of the function $I(C)$ provided we restrict ourselves to admissible arcs.

We shall have frequent occasion to use the integral

$$
L(C)=\int_{C} L(\dot{y}) d t=\int_{C}|\dot{y}| d t
$$

determining the length $L(C)$ of $C$. Similarly the symbol $H(C)$ will be used to denote a function of the form

$$
H(C)=\theta(a)+\int_{C} H(a, y, \dot{y}) d t
$$

where $\theta(a)$ is continuous and $H(a, y, p)$ is admissible. We shall be interested only in the case in which $H$ is $E$-dominated by $F$. Accordingly we shall suppose the constant $b$ and the set $\mathfrak{D}_{0}$ described above have been chosen so that

$$
\left|E_{H}(a, y, p, q)\right| \leqq b E_{F}(a, y, p, q)
$$

whenever $(a, y, p)$ is in $\mathfrak{D}_{0}$ and $(a, y, q)$ is in $\mathfrak{D}$.

Let $p^{i}(a, y)(i=0,1, \cdots, n)$ be a set of functions of class $C^{\prime}$ having $[a, y, p(a, y)]$ on $C_{0}$ when $(a, y)$ is on $C_{0}$ and satisfying the conditions 


$$
\phi^{\beta}[a, y, p(a, y)]=0 \quad(\beta=1, \cdots, m)
$$

on a neighborhood of $C_{0}$. With the help of these functions the function $J(C)$ can be written as the sum

$$
J(C)=J^{*}(C)+E_{F}^{*}(C)
$$

where

$$
\begin{aligned}
& J^{*}(C)=G(a)+\int_{C} F_{p^{i}}[a, y, p(a, y)] d y_{i}, \\
& E_{F}^{*}(C)=\int_{C} E_{F}[a, y, p(a, y), \dot{y}] d t .
\end{aligned}
$$

We propose to study the properties of $J(C)$ by studying the properties of $J^{*}(C)$ and $E_{F}^{*}(C)$. In like manner we have

$$
\begin{aligned}
& L(C)=L^{*}(C)+E_{L}^{*}(C), \\
& H(C)=H^{*}(C)+E_{H}^{*}(C) .
\end{aligned}
$$

In view of the relation (2.7) one has

$$
E_{L}^{*}(C) \leqq b E_{F}^{*}(C)
$$

for every arc $C$ in a neighborhood $\mathfrak{F}$ of $C_{0}$ in $a y$-space satisfying the differential equations $\phi^{\beta}=0$. Similarly if (2.15) holds as stated, we have

$$
\left|E_{H}^{*}(C)\right| \leqq b E_{F}^{*}(C)
$$

for every arc $C$ in $\mathfrak{F}$ along which $\phi^{\beta}=0$.

3. Three lemmas. An essential part of the results to be obtained is a direct consequence of the three lemmas to be described in this section. The first lemma is the following:

LEMMA 3.1. Let $\theta(a), B^{i}(a, y)$ be continuous functions and set

$$
H^{*}(C)=\theta(a)+\int_{C} B^{i}(a, y) d y^{i} .
$$

Given a constant $\epsilon^{\prime}>0$ there exists a constant $\delta>0$ and a neighborhood $\mathfrak{F}$ of $C_{0}$ in ay-space such that if $C_{1}$ is a subarc of $C_{0}$ the inequality

$$
\left|H^{*}(C)-H^{*}\left(C_{1}\right)\right|<\epsilon^{\prime}+\epsilon^{\prime} E_{F}^{*}(C)
$$

holds for every arc $C$ in $\mathfrak{F}$ satisfying $\phi^{\beta}=0$ and having its end values $\left[y\left(t^{1}\right), y\left(t^{2}\right)\right]$ in the $\delta$-neighborhood of those belonging to $C_{1}$. 
It is clear from continuity considerations that if the lemma is true when $\theta(a) \equiv 0$ it is true when $\theta(a) \not \equiv 0$. We shall accordingly assume that $\theta(a) \equiv 0$. Obviously we can assume that $a^{h}=0(h=1, \cdots, r)$ on $C_{0}$, that is, $a_{0}{ }^{h}=0$. In fact we can suppose that

$$
p^{0}(a, y)=1, \quad p^{i}(a, y)=0
$$

and that $y_{0} i(t) \equiv 0(i>0)$ on $C_{0}$. This result can be brought about by observing that a neighborhood $\mathfrak{F}$ of $C_{0}$ in $a y$-space can be simply covered by the solutions

$$
a^{h}, \quad y^{i}\left(t, a, b^{1}, \cdots, b^{n}\right)
$$

of the equations $\dot{y}^{i}=p^{i}(a, y)$. The family (3.3) contains $C_{0}$ for $(a, b)=(0,0)$ and has continuous derivatives with respect to $t, b^{1}, \cdots, b^{n}$ with a nonvanishing determinant. If $\mathfrak{F}$ is taken sufficiently small the variables $y^{0}, \cdots, y^{n}$ can be replaced by the new variables $\bar{y}^{0}=t, \bar{y}^{1}=b^{1}, \cdots, \bar{y}^{n}=b^{n}$. For these new variables $p^{0}=1, p^{1}=\cdots=p^{n}=0$ as desired. By virtue of Theorem 4.4 of the first paper, an inequality of the form (2.7) still holds.

Suppose now that the simplifications described in the last paragraph have been made and set $x=y^{0}$. The curves $C$ in which we are interested are then of the form

$$
a^{h}, \quad x(t), \quad y^{i}(t) \quad\left(t^{1} \leqq t \leqq t^{2} ; i=1, \cdots, n\right)
$$

and satisfy the differential equations $\phi^{\beta}(a, x, y, \dot{x}, \dot{y})=0$. Moreover $H^{*}(C)$ with $\theta(a) \equiv 0$ takes the form

$$
H^{*}(C)=\int_{C} B^{0}(a, x, y) d x+B^{i}(a, x, y) d y^{i} .
$$

The inequality (2.7) implies that

$$
\left(\dot{x}^{2}+\dot{y}^{i} \dot{y}^{i}\right)^{1 / 2}-\dot{x} \leqq b E_{F}(a, x, y, 1,0, \dot{x}, \dot{y})
$$

provided $\phi^{\beta}(a, x, y, \dot{x}, \dot{y})=0$. For an arc $C$ in a neighborhood $\mathfrak{F}$ of $C_{0}$ satisfying $\phi^{\beta}=0$ one has by integration

$$
L(C) \leqq x\left(t^{2}\right)-x\left(t^{1}\right)+b E_{F}^{*}(C) \leqq M+b E_{F}^{*}(C)
$$

where $M$ is chosen so that $x\left(t^{2}\right)-x\left(t^{1}\right)<M$. Given a constant $\epsilon^{\prime}>0$ choose a constant $\epsilon>0$ so that

$$
\epsilon(1+M)<\epsilon^{\prime}, \quad \epsilon b<\epsilon^{\prime} .
$$

We can suppose that $\mathfrak{F}$ has been chosen so small that the functions $B_{i}$ are defined and continuous on its closure. Then there exist functions $D^{i}(a, x)$ having continuous derivatives and satisfying on $\mathfrak{F}$ the condition

$$
\left|D^{i}(a, x)-B^{i}(a, x, 0)\right|<\epsilon / 4 n \quad(i=1, \cdots, n) .
$$


We now define (with $i=1, \cdots, n$ )

$$
\begin{aligned}
D^{0}(a, x, y) & =B^{0}(a, x, 0)+y^{i} \frac{\partial D^{i}}{\partial x}, \\
H_{1}(C) & =\int_{C} D^{0} d x+D^{i} d y^{i}, \\
H_{2}(C) & =\int_{C}\left(B^{0}-D^{0}\right) d x+\left(B^{i}-D^{i}\right) d y^{i}
\end{aligned}
$$

so that

$$
H^{*}(C)=H_{1}(C)+H_{2}(C) .
$$

By virtue of the definitions of the functions $D^{0}(a, x, y), D^{i}(a, x, y)=D^{i}(a, x)$ $(i=1, \cdots, n)$ we can diminish the neighborhood $\mathfrak{F}$ of $C_{0}$ still more so that the inequalities

$$
\left|B^{k}(a, x, y)-D^{k}(a, x, y)\right|<\epsilon / 2 n \quad(k=0,1, \cdots, n)
$$

hold on $\mathfrak{F}$. Consequently the relation

$$
\left|\left(B^{0}-D^{0}\right) \dot{x}+\left(B^{i}-D^{i}\right) \dot{y}^{i}\right|<\epsilon\left(\dot{x}^{2}+\dot{y}^{i} \dot{y}^{i}\right)^{1 / 2}
$$

is also valid on $\mathfrak{F}$ and hence one has

$$
\left|H_{2}(C)\right|<\epsilon L(C)
$$

for every arc $C$ in $\mathfrak{F}$. By construction one has

$$
\partial D^{0} / \partial y^{i}=\partial D^{i} / \partial x, \quad \partial D^{i} / \partial y^{k}=0 \quad(i, k=1, \cdots, n)
$$

on $\mathfrak{F}$. It follows that $H_{1}(C)$ is an invariant integral and is accordingly a continuous function of the end values $\left(a, x^{1}, y^{1}, x^{2}, y^{2}\right)$ of $C$. If we regard points of $C_{0}$ as subarcs of $C_{0}$ there is, by continuity, a constant $\delta>0$. so small that the relation

$$
\left|H_{1}(C)-H_{1}\left(C_{1}\right)\right|<\epsilon
$$

holds for every pair of arcs $C_{1}, C$ such that $C_{1}$ is a subarc of $C_{0}$ and the end values of $C$ are in the $\delta$-neighborhoods of those of $C_{1}$. Consider now a subarc $C_{1}$ of $C_{0}$ and let $C$ be an arc along which $\phi^{\beta}=0$ and having its end values in the $\delta$-neighborhood of those of $C_{1}$. Using the relations

$$
\left|H^{*}(C)-H^{*}\left(C_{1}\right)\right| \leqq\left|H_{1}(C)-H_{1}\left(C_{1}\right)\right|+\left|H_{2}(C)\right|
$$

which hold because of the relations (3.6) and $H_{2}\left(C_{1}\right)=0$, it is found by the use of (3.8), (3.7), (3.4) that

$$
\left|H^{*}(C)-H^{*}\left(C_{1}\right)\right| \leqq \epsilon+\epsilon L(C) \leqq \epsilon(1+M)+\epsilon b E_{F}^{*}(C) .
$$


This result combined with (3.5) gives us the desired relation (3.2) provided we diminish $\mathfrak{F}$ so that $|a|<\delta$ in $\mathfrak{F}$.

LemMA 3.2. Given a constant $\epsilon^{\prime}>0$ there exists a constant $\delta>0$ and a 'neighborhood $\mathfrak{F}$ of $C_{0}$ in ay-space such that if $C_{1}$ is a subarc of $C_{0}$ and $C$ is an arc in $\mathfrak{F}$ along which $\phi^{\beta}=0$ and having its ends in the $\delta$-neighborhood of those on $C_{1}$, then

$$
-\epsilon^{\prime}+\left(1-\epsilon^{\prime}\right) E_{F}^{*}(C) \leqq J(C)-J\left(C_{1}\right) \leqq \epsilon^{\prime}+\left(1+\epsilon^{\prime}\right) E_{F}^{*}(C) .
$$

For let $\delta, \mathfrak{F}$ be related to $\epsilon^{\prime}$ as described in the last lemma with $H^{*}(C)$ $=J^{*}(C)$, where $J^{*}(C)$ is given by (2.18). The lemma now follows from Lemma 3.1 and the relation

$$
J(C)-J\left(C_{1}\right)=J^{*}(C)-J^{*}\left(C_{1}\right)+E_{F}^{*}(C)
$$

which holds by virtue of $(2.17)$ and the relation $E_{F}{ }^{*}\left(C_{1}\right)=0$.

Lemma 3.3. Let $H(C)$ be defined by (2.14) and suppose $H$ is E-dominated by $F$ near $C_{0}$ on $\mathfrak{D}$. Let $b$ be a constant effective as in (2.15). Given a constant $\epsilon^{\prime}>0$ there exists a constant $\delta>0$ and $a$ neighborhood $\mathfrak{F}$ of $C_{0}$ in ay-space such that the inequality

$$
\left|H(C)-H\left(C_{1}\right)\right| \leqq \epsilon^{\prime}+\left(b+\epsilon^{\prime}\right) E_{F}^{*}(C)
$$

holds when $C_{1}$ is a subarc of $C_{0}$ and $C$ is an arc in $\mathfrak{F}$ with its end values in the $\delta$-neighborhood of those of $C_{1}$ and along which $\phi^{\beta}=0$.

This result follows from Lemma 3.1 with the help of (2.22) and the relation

$$
H(C)-H\left(C_{1}\right)=H^{*}(C)-H^{*}\left(C_{1}\right)+E_{H}^{*}(C) .
$$

4. An analogue of the theorem of Lindeberg. The following two theorems are basic. They are essentially extensions of similar theorems given by Reid( $\left.{ }^{(}\right)$ for the nonparametric case. They differ from those given by Reid in that the neighbarhood $\mathfrak{F}$ and constants $\rho, \delta$ can be chosen so as to apply uniformly for all subarcs $C_{1}$ of $C_{0}$.

THEOREM 4.1. Suppose that $H(a, y, p)$ is E-dominated by $F(a, y, p)$ near $C_{0}$ on $\mathfrak{D}$. Given a constant $\epsilon>0$ there exist positive constants $\delta, \rho$ and a neighborhood $\mathfrak{F}$ of $C_{0}$ in ay-space such that given a subarc $C_{1}$ of $C_{0}$, the inequality

$$
\left|H(C)-H\left(C_{1}\right)\right|<\epsilon
$$

holds for every arc $C$ in $\mathfrak{F}$ satisfying the differential equations $\phi^{\beta}=0$, the inequality

$$
E_{F}^{*}(C) \leqq \rho,
$$

and having its end values $\left[y\left(t^{1}\right), y\left(t^{2}\right)\right]$ in the $\delta$-neighborhood of those on $C_{1}$.

(8) W. T. Reid, loc. cit. \$4. 
In order to prove this result let $b$ be a constant effective as in (2.15) and choose positive constants $\epsilon^{\prime}, \rho$ such that

$$
\epsilon^{\prime}+\left(b+\epsilon^{\prime}\right) \rho<\epsilon .
$$

Choose $\mathfrak{F}$ and $\delta$ related to $\epsilon^{\prime}$ and $C_{0}$ as described in Lemma 3.3. The relation (4.1) then follows from (4.2), (3.11), and (4.3).

As a second result we have the following analogue of the theorem of Lindeberg.

ThEOREM 4.2. Given a constant $\eta>0$ there exist positive constants $\delta, \rho_{1}$ and a neighborhood $\mathfrak{F}$ of $C_{0}$ in ay-space such that the following statements are true: Let $C_{1}$ be $a$ subarc of $C_{0}$ and let $C$ be an arc in $\mathfrak{F}$ along which $\phi^{\beta}=0$ and having its end values $\left[y\left(t^{1}\right), y\left(t^{2}\right)\right]$ in the $\delta$-neighborhoods of those on $C_{1}$. Then

$$
J(C)>J\left(C_{1}\right)-\eta \text {. }
$$

If $C$ satisfies the condition (4.2) with $\rho=\rho_{1}$ we have

$$
J(C)<J\left(C_{1}\right)+\eta \text {. }
$$

If $C$ satisfies the condition

$$
E_{F}^{*}(C) \geqq 2 \eta,
$$

then

$$
J(C)>J\left(C_{1}\right)+n .
$$

In order to prove this result select $\epsilon^{\prime}$ and $\rho_{1}$ to be positive constants satisfying the following conditions

$$
\epsilon^{\prime}<\eta, \quad \eta<-\epsilon^{\prime}+\left(1-\epsilon^{\prime}\right) 2 \eta, \quad \epsilon^{\prime}+\left(1+\epsilon^{\prime}\right) \rho_{1}<\eta .
$$

Choose $\mathfrak{F}$ and $\delta$ related to $\epsilon^{\prime}$ and $C_{0}$ as described in Lemma 3.2. The relation (4.4) follows from (3.9) since $\epsilon^{\prime}<\eta, \epsilon^{\prime}<1$, and $E_{F}^{*}(C) \geqq 0$. The relation (4.5) follows from (4.2) and the last inequalities in (4.8) and (3.9). Finally (4.7) follows from (4.6), (3.9), and the second inequality (4.8).

5. Consequences of the theorem of Lindeberg. The results described in the last section have many important consequences. One of the most significant of these consequences is given in the following theorem.

TheOREM 5.1 $\left({ }^{7}\right)$. Given a constant $\epsilon>0$ there exist positive constants $\delta, \eta$ and

( ${ }^{7}$ ) Added in proof. This theorem is a consequence of the following more general result which follows readily from the inequality (4.4) and the concept of $E$-dominance.

Theorem. If $L$ and $H$ are $E$-dominated by $F$ near $C_{0}$ on $\mathfrak{D}$, there is a positive constant $b$ such that given a constant $\eta>0$ there is a constant $\delta>0$ and a neighborhood $\varsubsetneqq$ of $C_{0}$ in ay-space such that if $C_{1}$ is a subarc of $C_{0}$ the inequality $J(C)-J\left(C_{1}\right) \geqq b\left|H(C)-H\left(C_{1}\right)\right|-\eta$ holds for every arc $C$ in $\mathfrak{F}$ satisfying the differential equations $\phi^{\beta}=0$ and having its end values in the $\delta$-neighborhood of those on $C_{1}$. 
a neighborhood $\mathfrak{F}$ of $C_{0}$ in ay-space such that if $C_{1}$ is a subarc of $C_{0}$ the inequality

$$
\left|H(C)-H\left(C_{1}\right)\right|<\epsilon
$$

holds for every arc $C$ in $\mathfrak{F}$ satisfying the differential equations $\phi^{\beta}=0$, the inequality

$$
J(C) \leqq J\left(C_{1}\right)+\eta
$$

and having its end values $\left[y\left(t^{1}\right), y\left(t^{2}\right)\right]$ in the $\delta$-neighborhood of those of $C_{1}$.

For let $\mathfrak{F}, \delta, \rho$ be related to $\epsilon$ and $H(C)$ as described in Theorem 4.1. Diminish $\mathfrak{F}$ and $\delta$ so that they are related to $\eta=\rho / 2$ and $J(C)$ as described in Theorem 4.2. Consider now an arc $C$ related to a subarc $C_{1}$ of $C_{0}$ as described in the theorem. If the relation (5.1) failed to hold, then, by Theorem 4.1 , we would have

$$
E_{F}^{*}(C)>\rho=2 \eta \text {. }
$$

By Theorem 4.2 the relation (4.7) would hold, contrary to our assumption that (5.2) held. This proves the theorem.

The last theorem can be restated as described in the following corollary.

Corollary. Given a constant $\epsilon>0$ there exist positive constants $\delta, \eta$ and $a$ neighborhood $\mathfrak{F}$ of $C_{0}$ in ay-space such that, if $C_{1}$ is a subarc of $C_{0}$, the inequality

$$
J(C)>J\left(C_{1}\right)+\eta
$$

holds for every arc $C$ in $\mathfrak{F}$ satisfying the differential equations $\phi^{\beta}=0$, the inequality

$$
\left|H(C)-H\left(C_{1}\right)\right| \geqq \epsilon,
$$

and having its end values $\left[y\left(t^{1}\right), y\left(t^{2}\right)\right]$ in the $\delta$-neighborhood of the end values of $C_{1}$.

In case we require the subarc $C_{1}$ of $C_{0}$ described in Theorem 5.1 to be the whole arc $C_{0}$ and we require the arc $C$ to satisfy the end conditions (2.2) so as to be admissible, then the region $\mathfrak{F}$ described in Theorem 5.1 can be taken so small that the end values of $C$ are in the $\delta$-neighborhood of the end values of $C_{0}$ prescribed in Theorem 5.1. Moreover $J(C)=l^{0} I(C)$ and $J\left(C_{0}\right)$ $=l^{\circ} I\left(C_{0}\right)$. By virtue of Theorem 5.1 we have accordingly the following result. Recall that an admissible arc is one that satisfies equations $(2.1),(2.2)_{2}$ and (2.3).

TheOREM 5.2. Given a constant $\epsilon>0$ there exist a constant $\eta>0$ and $a$ neighborhood $\mathfrak{F}$ of $C_{0}$ in ay-space such that the inequality

$$
\left|H(C)-H\left(C_{0}\right)\right|<\epsilon
$$

holds, for every admissible arc $C$ in $\mathfrak{F}$ satisfying the conditions 


$$
J(C)=l^{0} I(C) \leqq l^{0} I\left(C_{0}\right)+\eta=J\left(C_{0}\right)+\eta .
$$

In case $l^{0}=0$ the inequality (5.6) is always satisfied. Hence we have the following corollary.

CoRollary. Suppose the constant $l^{\circ}$ appearing in the definition of $J(C)$ is zero. Then given a constant $\epsilon>0$ there exists a neighborhood $\mathfrak{F}$ of $C_{0}$ in ay-space such that the inequality (5.5) holds for every admissible arc $C$ in $\mathfrak{F}$.

By taking $H(C)=L(C)$ in Theorem 5.2 one obtains readily the further results for arcs satisfying equations (2.1), (2.2), and (2.3).

THEOREM 5.3. Suppose there exists a neighborhood $\mathfrak{F}_{1}$ of $C_{0}$ in ay-space and a constant $\epsilon>0$ such that the inequality $J(C)>J\left(C_{0}\right)$ holds for every admissible $\operatorname{arc} C \neq C_{0}$ in $\mathfrak{F}_{1}$ satisfying the condition

$$
\left|L(C)-L\left(C_{0}\right)\right|<\epsilon,
$$

then there exists a neighborhood $\mathfrak{F}$ of $C_{0}$ in ay-space such that the inequality $J(C)>J\left(C_{0}\right)$ holds for every admissible arc $C \neq C_{0}$ in $\mathfrak{F}$.

6. A remark concerning sufficiency theorems. Let $C^{*}$ be any arc which does not intersect itself, satisfies the differential equations $\phi^{\beta}=0$ and contains $C_{0}$ as a subarc. If $F E$-dominates $L$ near $C^{*}$ on $\mathfrak{D}$ and $C_{0}$ is a minimizing arc for the problem formulated in $\$ 2$ then there is a neighborhood $\mathfrak{F}$ on $C^{*}$ such that $J(C) \geqq J\left(C_{0}\right)$ for every admissible arc $C$ in $\mathfrak{F}$. This result is given in the following theorem. One should observe that the arc $C^{*}$ need only satisfy the conditions described above and can differ appreciably from $C_{0}$ not only in length but in that, apart from the subarc $C_{0}$, it need not satisfy the EulerLagrange equations associated with our problem. As before, admissible arcs are those that satisfy equations (2.1), (2.2), and (2.3).

Theorem 6.1. Let $C^{*}$ be an arc having the properties described above and suppose that $L=|p|$ is $E$-dominated near $C^{*}$ on $\mathfrak{D}$ by the integrand $F$ of $J(C)$. If there is a neighborhood $\mathfrak{F}_{1}$ of $C_{0}$ such that $J(C)>J\left(C_{0}\right)$ for every admissible arc $C \neq C_{0}$ in $\mathfrak{F}_{1}$, then there is a neighborhood $\mathfrak{F}$ of $C^{*}$ such that $J(C)>J\left(C_{0}\right)$ for every admissible arc $C \neq C_{0}$ in $\mathfrak{~}$.

In order to prove this result choose a constant $\epsilon>0$ and a neighborhood $\mathfrak{F}$ of $C^{*}$ so small that an admissible arc in $\mathfrak{F}$ satisfying the condition

$$
\left|L(C)-L\left(C_{0}\right)\right|<\epsilon
$$

must be in $\mathfrak{F}_{1}$. Let $C^{*}$ play the role of $C_{0}$ in Theorem 5.1 and select $H(C)$ $=L(C)$. Then by Theorem 5.1 with $C_{0}, C_{1}$ replaced by $C^{*}, C_{0}$ we can select constants $\delta, \eta$ and diminish $₹$ so that the inequality (6.1) holds for every admissible arc $C$ in $\mathfrak{F}$ satisfying the condition

$$
J(C) \leqq J\left(C_{0}\right)+\eta
$$


and having the end values of $C$ in the $\delta$-neighborhood of those of $C_{0}$. By virtue of the continuity of the end conditions (2.2) we can diminish $\mathfrak{F}$ further so that the end values of every admissible arc $C$ in $\mathfrak{F}$ lie in the $\delta$-neighborhood of those belonging to $C_{0}$. If an admissible arc $C$ in $\mathfrak{F}$ satisfies the condition (6.2), then (6.1) holds by Theorem 5.1. By virtue of our choice of $\epsilon$ and $\mathfrak{F}$ it follows that $C$ is in $\mathfrak{F}_{1}$ and hence that $J(C)>J\left(C_{0}\right)$ provided $C \neq C_{0}$. From this result it is seen that we have $J(C)>J\left(C_{0}\right)$ for every admissible arc $C \neq C_{0}$ in $\mathfrak{F}$.

It is interesting to observe that although the hypotheses of our theorem require that the arc $C_{0}$ be an extremal, the extension $C^{*}$ of $C$ need not be an extremal. An extremal is an arc of class $C^{\prime \prime}$ satisfying the Euler-Lagrange differential equations

$$
\frac{d}{d t} F_{p^{i}}=F_{y^{i}}, \quad \phi^{\beta}=0 .
$$

In the following corollary the $\operatorname{arcs} C_{1}, C_{0}$ play the roles of $C_{0}, C^{*}$ in Theorem 6.1.

Corollary. Let $C_{1}$ be a subarc of $C_{0}$ and suppose there is a neighborhood $\mathfrak{F}_{1}$ of $C_{1}$ in ay-space such that the inequality $J(C)>J\left(C_{1}\right)$ holds for every arc $C \neq C_{1}$ in $\mathfrak{F}_{1}$ having the same end values $\left[a, y\left(t^{1}\right), y\left(t^{2}\right)\right]$ as $C_{1}$ and satisfying the differential equations $\phi^{\beta}=0$. There exists a neighborhood $\mathfrak{F}$ of $C_{0}$ in ay-space such that the inequality $J(C)>J\left(C_{1}\right)$ holds for every arc $C \neq C_{1}$ in $\mathfrak{F}$ having the same end values $\left[a, y\left(t^{1}\right), y\left(t^{2}\right)\right]$ as $C_{1}$ and satisfying the differential equations $\phi^{\beta}=0$.

The hypotheses in the corollary are satisfied when $C_{1}$ is an extremal for $J(C)$ having on it no pairs of conjugate points. In fact the result still holds in this case when $C_{1}$ is replaced by a neighboring extremal as described in the following theorem.

THEOREM 6.2. Let $C_{1}$ be a subarc of $C_{0}$ and suppose that $C_{1}$ is an extremal for $J(C)$ having on it no pairs of conjugate point's relative to the class of differentially admissible arcs. There exist a constant $\delta>0$ and a neighborhood $\mathfrak{F}$ of $C_{0}$ such. that if $C_{2}$ is an extremal in $\mathfrak{F}$ with end values $\left[a, y\left(t^{1}\right), y\left(t^{2}\right)\right]$ in the $\delta$-neighborhood of those on $C_{1}$, the inequality $J(C)>J\left(C_{2}\right)$ holds for every arc $C \neq C_{2}$ in $\mathfrak{F}$ whose end values $\left[a, y\left(t^{1}\right), y\left(t^{2}\right)\right]$ coincide with those on $C_{2}$ and along which $\phi^{\beta}=0$.

The complete proof of this result will not be given here. The theorem can be established by an argument like that used in the proof of the last theorem together with an application of Hahn's Lemma. This lemma states that if $C_{1}$ is a nonsingular extremal satisfying the condition of Weierstrass and having on it no pairs of conjugate points, there is a constant $\delta>0$ and a neighborhood $\mathfrak{F}_{1}$ of $C_{1}$ such that if $C_{2}$ is an extremal in $\mathfrak{F}_{1}$ with end values $\left[a, y\left(t^{1}\right), y\left(t^{2}\right)\right]$ in the $\delta$-neighborhood of those on $C_{1}$, then the inequality $J(C)>J\left(C_{2}\right)$ holds for every differentially admissible $\operatorname{arc} C \not C_{2}$ in $\mathfrak{F}_{1}$ having the same end values 
$\left[a, y\left(t^{1}\right), y\left(t^{2}\right)\right]$ as $C_{2}$. This lemma can be established by an argument like that used by Bliss $\left.{ }^{8}\right)$ for the nonparametric problem of Bolza.

Theorem 6.2 is an extension to the problem of Bolza of a result given by Birkhoff and Hestenes $\left({ }^{9}\right)$ and later by Karush $\left({ }^{10}\right)$. The method here outlined is simpler than the ones used previously.

Theorem 6.3. Suppose that $C_{0}$ is an extremal. There exist successive points $P_{1}, \cdots, P_{k}$ on $C_{0}$ and a neighborhood of $C_{0}$ in ay-space such that the inequality $J(C)>J\left(C_{0}\right)$ holds for every arc $C \neq C_{0}$ in $\mathfrak{F}$ satisfying $\phi^{\beta}=0$, having the same end values $\left[a, y\left(t^{1}\right), y\left(t^{2}\right)\right]$ as $C_{0}$, and having $P_{1}, \cdots, P_{k}$ as successive points on $C$.

To prove this result select the points $P_{1}, \cdots, P_{k}$ so as to divide $C_{0}$ into subarcs that are minimizing arcs in the class of subarcs joining its end points and satisfying $\phi^{\beta}=0$. This can be done because the distance between pairs of points conjugate to each other has a positive lower bound. The theorem then follows readily from Theorem 6.1 applied to each subarc of $C_{0}$.

The result described in Theorem 6.3 is not the most general theorem of this type that can be stated. In order to obtain these generalizations one is led to the study of index theorems. These theorems will not be developed in this paper.

7. Further consequences of the theorem of Lindeberg. Many of the applications of the theorem of Lindeberg are consequences of the following analogue of Theorem 5.1.

TheOREM 7.1. Given a constant $\epsilon>0$ there exist positive constants $\delta, \eta$ and a neighborhood $\mathfrak{F}$ of $C_{0}$ in ay-space such that if $C$ is an admissible arc in $\mathfrak{F}$ satisfying the condition

$$
J(C) \leqq J\left(C_{0}\right)+\eta
$$

the inequality

$$
\left|H\left(C^{\prime}\right)-H\left(C_{1}\right)\right|<\epsilon
$$

will hold if $C_{1}$ is a subarc of $C_{0}$ and $C^{\prime}$ is a subarc of $C$ having its end values in the $\delta$-neighborhood of those on $C_{1}$.

For choose $\mathfrak{F}, \delta, \rho, \eta=\rho / 2$ related to $\epsilon$ as described in the proof of Theorem 5.1. Choose $C_{1}, C, C^{\prime}$ as described in Theorem 7.1. If the inequality (7.2) failed to hold, then we would have $E_{F}{ }^{*}\left(C^{\prime}\right)>\rho=2 \eta$, by Theorem 4.1. Since

(8) G. A. Bliss, The problem of Bolza in the calculus of variations, Ann. of Math. (2) vol. 33 (1932) pp. 261-274.

(') G. D. Birkhoff and M. R. Hestenes, Natural isoperimetric conditions in the calculus of variations, Duke Math. J. vol. 1 (1935) pp. 198-286.

$\left.{ }^{(10}\right)$ W. Karush, Isoperimetric problems and index theorems in the calculus of variations, Dissertation, The University of Chicago, 1940. 
$E_{F}$ is non-negative along $C$ we would have $E_{F}{ }^{*}(C) \geqq E_{F}^{*}\left(C^{\prime}\right)>2 \eta$. By Theorem 4.2 we would have $J(C)>J\left(C_{0}\right)+\eta$, contrary to our assumption that (7.1) held. This proves the theorem.

One of the immediate applications of the last theorem is to be found in the next theorem. In this theorem the function $H^{k}(C)$ is of the form

$$
H^{k}(C)=\theta^{k}(a)+\int_{t^{2}}^{t^{2}} H^{k}(a, y, \dot{y}) d t \quad(k=1, \cdots, q)
$$

where $\theta^{k}(a)$ is continuous on $\Re$ and $H^{k}(a, y, p)$ is an admissible function that is $E$-dominated by $F$ near $C_{0}$ on $\mathfrak{D}$. With each admissible arc

C:

$$
a^{h}, \quad y^{i}(t)
$$

we associate a set of $q$ functions $z^{k}(t)$ defined by the equations

$$
z^{k}(t)=\theta^{k}(a)+\int_{t^{1}}^{t} H^{k}[a, y(t), \dot{y}(t)] d t \quad(k=1, \cdots, q) .
$$

We can now prove the following theorem.

Theorem 7.2. Let $\mathfrak{M}$ be a neighborhood of the elements $(a, y, z)$ on $C_{0}$. Given $a$ constant $\epsilon>0$ there exists a constant $\eta>0$ and $a$ neighborhood $\mathfrak{F}$ of $C_{0}$ in ay-space such that if $C$ is an admissible arc in $\mathfrak{F}$ satisfying the condition

$$
J(C) \leqq J\left(C_{0}\right)+\eta
$$

then the elements $(a, y, z)$ on $C$ are in $\mathfrak{M}$ and

$$
\left|H^{k}(C)-H^{k}\left(C_{0}\right)\right|<\epsilon \quad(k=1, \cdots, q) .
$$

In order to prove this result we may suppose that $\epsilon$ has been chosen so that an element $(a, y, z)$ in the $2 q \epsilon$-neighborhood of those on $C_{0}$ is in $\mathfrak{M}$. Choose $F, \eta, \delta(\delta<\epsilon)$ related to $\epsilon$ and each of the functions $H(C)=H^{k}(C)$ as described in Theorem 7.1. Diminish $\mathfrak{F}$ so that it is in the $\delta$-neighborhood of the points $(a, y)$ on $C_{0}$ and so that the end values of every admissible arc $C$ in $\mathfrak{F}$ are in the $\delta$-neighborhoods of those on $C_{0}$. Consider now an element $(\bar{a}, \bar{y}, \bar{z})$ on an admissible $\operatorname{arc} C$ in $\mathfrak{F}$. Let $C^{\prime}$ be the subarc of $C$ joining the initial point of $C$ to the point $(\bar{a}, \bar{y})$ on $C$. Then $\bar{z}^{k}=H^{k}\left(C^{\prime}\right)$. By virtue of our choice of $\mathfrak{F}$ there is a point $\left(\bar{a}_{0}, \bar{y}_{0}\right)$ on $C_{0}$ in the $\delta$-neighborhood of $(\bar{a}, \bar{y})$. Let $C_{1}$ be the subarc of $C_{0}$ joining the initial point of $C_{0}$ to the point $\left(\bar{a}_{0}, \bar{y}_{0}\right)$. Then the element $\left(\bar{a}_{0}, \bar{y}_{0}, \bar{z}_{0}\right)$ on $C_{0}$ determined by $\left(\bar{a}_{0}, \bar{y}_{0}\right)$ is such that $\bar{z}_{0}{ }^{k}=H^{k}\left(C_{1}\right)$. If $C$ satisfies the condition (7.4) we have, by Theorem 7.1,

$$
\left|\bar{z}^{k}-\bar{z}_{0}^{k}\right|=\left|H^{k}\left(C^{\prime}\right)-H^{k}\left(C_{1}\right)\right|<\epsilon \quad(k=1, \cdots, q) .
$$

Since $\delta<\epsilon$, this result implies that the element $(\bar{a}, \bar{y}, \bar{z})$ is in the $2 q \epsilon$-neighborhood of the element $\left(\bar{a}_{0}, \bar{y}_{0}, \bar{z}_{0}\right)$ on $C_{0}$ and hence is in $\mathfrak{M}$. This proves the theorem. 
COROLlaRy 1. If there exists a neighborhood $\mathfrak{M}$ of the elements $(a, y, z)$ on $C_{0}$ and a constant $\epsilon>0$ such that the inequality $J(C)>J\left(C_{0}\right)$ holds for every admissible arc $C \neq C_{0}$ which satisfies (7.5) and whose elements $(a, y, z)$ are in $\mathfrak{M}$, then there exists a neighborhood $\mathfrak{F}$ of the points $(a, y)$ on $C_{0}$ such that the inequality $J(C)>J\left(C_{0}\right)$ holds for every admissible arc $C \neq C_{0}$ in $\mathfrak{F}$.

This result is equivalent to the further corollary:

COROLlARY 2. Let $b^{k}$ be the value of $z^{k}\left(t^{2}\right)$ on an arc $C$. If there exists a neighborhood $\mathfrak{N}$ of $C_{0}$ in $(a, b, y, z)$-space such that the inequality $J(C)>J\left(C_{0}\right)$ holds for every admissible arc $C \neq C_{0}$ whose elements $(a, b, y, z)$ are in $\mathfrak{N}$, then there exists a neighborhood $\mathfrak{F}$ of $C_{0}$ in ay-space such that the inequality $J(C)>J\left(C_{0}\right)$

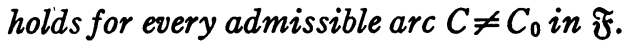

8. Isoperimetric versus non-isoperimetric problems. The result obtained in the last section can be used to show that the sufficiency theorems for the isoperimetric problems can be obtained from those for the non-isoperimetric problems. To this end observe that if one sets

$$
z^{\sigma}(t)=g^{\sigma}(a)+\int_{t^{1}}^{t} f^{\sigma}[a, y(t), \dot{y}(t)] d t \quad(\sigma=1, \cdots, s)
$$

then the problem here given is equivalent to finding in the class of arcs

$$
a^{h}, \quad y^{i}(t), \quad z^{\sigma}(t) \quad\left(t^{1} \leqq t \leqq t^{2}\right)
$$

satisfying the conditions

$$
\begin{gathered}
\phi^{\beta}(a, y, \dot{y})=0, \quad \dot{z}^{\sigma}-f^{\sigma}(a, y, \dot{y})=0, \\
y^{i}\left(t^{1}\right)=T^{i 1}(a), \quad z^{\sigma}\left(t^{1}\right)=g^{\sigma}(a), \quad y^{i}\left(t^{2}\right)=T^{i 2}(a), \quad z^{\sigma}\left(t^{2}\right)=0
\end{gathered}
$$

one which minimizes

$$
I(C)=g(a)+\int_{t^{1}}^{t^{2}} f(a, y, \dot{y}) d t .
$$

This problem is a non-isoperimetric problem. The sufficiency conditions, as usually stated, are equivalent for the two problems. However neighborhoods are not equivalent since given a neighborhood $\mathfrak{M}$ of $C_{0}$ in ayz-space it is not in general possible to find a neighborhood $\mathfrak{F}$ of $C_{0}$ in $a y$-space such that every admissible arc $C$ in $\mathfrak{F}$ has its elements $(a, y, z)$ in $\mathfrak{M}$. Thus the concept of $a$ proper strong relative minimum is not the same for the two problems. The gap between these two concepts is bridged by the results described in the last section. Under the sufficiency theorem, as usually stated, one can be assured, by the theory for the non-isoperimetric problem of Bolza, that there exists a neighborhood $\mathfrak{M}$ of $C_{0}$ in ayz-space such that the inequality

$$
J(C)=l^{0} I(C)>J\left(C_{0}\right)=l^{0} I\left(C_{0}\right)
$$


holds for every admissible arc $C \neq C_{0}$ with elements $(a, y, z)$ in $\mathfrak{M}$. Moreover these conditions imply that the hypotheses on which Theorem 7.2 is based are satisfied. It follows from the corollary to Theorem 7.2 that there is a neighborhood $\mathfrak{F}$ of $C_{0}$ in $a y$-space such that the inequality (8.1) holds for every admissible arc $C \neq C_{0}$ in $\mathfrak{F}$. It is clear from (8.1) that if our problem is to be nontrivial the multiplier $l^{0}$ must be different from zero and hence can be taken to be unity. The method of proof just described is an obvious extension to parametric problems of a similar method used by Reid for nonparametric problems.

9. Parametric problems versus nonparametric problems. The purpose of the present section is to show that the sufficiency theorems for the parametric problems can be obtained from those for the nonparametric problems by the use of Theorem 7.2. To this end let $H(a, y, p)$ be an admissible function of class $C^{\prime \prime}$ that is positive on $\Re$ and that is $E$-dominated by $F$ near $C_{0}$ on $\mathfrak{D}$. For example, $H=L(p)=|p|$ is such a function. If $p^{0}>0$ for every element $(x, y, p)$ in $\Re$, then $H=p^{0}$ also has this property. The latter case arises when the problem at hand is already a nonparametric problem but is phrased as a parametric one.

The problem at hand is unaltered if we require the parameter $t$ of our arcs to be chosen so that the conditions

$$
H[a, y(t), \dot{y}(t)]-1=0, \quad t^{1}=0
$$

are satisfied. The parameter $t$ for each arc is then uniquely determined. If we set $b=t^{2}$, then on each $\operatorname{arc} C$ we have the set of

$$
a, \quad b, \quad t ; \quad y(t)
$$$$
\left(t^{1} \leqq t \leqq t^{2}\right)
$$

in $(a, b, t, y)$-space. Our problem is therefore equivalent to that of finding among arcs $C$ of the form (9.1) satisfying the conditions

$$
\begin{gathered}
\phi^{0}=H(a, y, \dot{y})-1=0, \quad \phi^{\phi}(a, y, \dot{y})=0, \\
t^{1}=0, \quad y^{i}\left(t^{1}\right)=T^{i 1}(a), \quad t^{2}=b, \quad y^{i}\left(t^{2}\right)=T^{i 2}(a), \\
I^{\sigma}(C)=g^{\sigma}(a)+\int_{t^{1}}^{t^{2}} f^{\sigma}(a, y, \dot{y}) d t=0
\end{gathered}
$$

one which minimizes the integral

$$
I(C)=g(a)+\int_{t^{1}}^{t^{2}} f(a, y, \dot{y}) d t .
$$

We shall suppose that $C_{0}$ is such an arc. Then there is a neighborhood $\mathfrak{N}$ of $C_{0}$ in $(a, b, t, y)$-space such that the inequality

$$
I(C)>I\left(C_{0}\right)
$$


holds for every (nonparametric) admissible arc $C \neq C_{0}$ whose elements $(a, b, t, y)$ are in $\mathfrak{R}$. We make the additional assumption that $l^{0} \neq 0$. We can accordingly suppose $l^{\circ}=1$. Then $(9.2)$ is equivalent to the condition

$$
J(C)>J\left(C_{0}\right) \text {. }
$$

Setting

$$
z(t)=\int_{t^{1}}^{t} H(a, y, \dot{y}) d t
$$

it is seen that when $H=1$ and $t^{1}=0$ we have $z=t$. The hypotheses of Corollary 2 to Theorem 7.2 are accordingly satisfied. There exists accordingly a neighborhood $\mathfrak{F}$ of $C_{0}$ in $a y$-space such that the inequality (9.3) and hence also (9.2) holds for every admissible arc $C \neq C_{0}$ in $\mathfrak{F}$, as was to be proved.

10. The problem of Mayer versus the problem of Bolza. In the present section we shall assume, for convenience, that $l^{0}=1$. It is our purpose to show that in this event the sufficiency theorems for the problem of Bolza are a consequence of those for the problem of Mayer. The problem of Mayer is the special case of the problem here considered in which $f \equiv f^{\circ} \equiv 0$. As is well known the problem here considered is equivalent to the following problem of Mayer: Given a class of arcs

$$
C: \quad a^{h}, \quad b^{k}, \quad y^{i}(t), \quad z^{k}(t) \quad\left(t^{1} \leqq t \leqq t^{2} ; k=0,1, \cdots, s\right)
$$

satisfying the conditions

$$
\begin{aligned}
& \phi^{\beta}(a, y, \dot{y})=0 \\
& (\beta=1, \cdots, m), \\
& f(a, y, \dot{y})-\dot{z}^{0}=0, \quad f^{\sigma}(a, y, \dot{y})-\dot{z}^{\sigma}=0 \quad(\sigma=1, \cdots, s), \\
& y^{i}\left(t^{1}\right)=T^{i 1}(a), \quad z^{k}\left(t^{1}\right)=0, \quad y^{i}\left(t^{2}\right)=T^{i 2}(a), \quad z^{k}\left(t^{2}\right)=b^{k}, . \\
& I^{\sigma}(C)=g^{\sigma}(a)+b^{\sigma}=0 \\
& (\sigma=1, \cdots, s)
\end{aligned}
$$

to find one which minimizes the sum

$$
I(C)=g(a)+b^{0} .
$$

The equivalence of this problem with the problem described in $\$ 2$ is seen at once if one observes that

$$
\begin{aligned}
z^{0}(t) & =\int_{t^{1}}^{t} f d t, & z^{\sigma}(t) & =\int_{t^{1}}^{t} f^{\sigma} d t, \\
b^{0} & =\int_{t^{1}}^{t^{2}} f d t, & b^{\sigma} & =\int_{t^{1}}^{t^{2}} f^{\sigma} d t .
\end{aligned}
$$

We shall suppose that under this transformation $C_{0}$ is a minimizing arc for the problem of Mayer just described. Then there is a neighborhood $\mathfrak{N}$ of $C_{0}$ in $(a, b, y, z)$-space such that the inequality 


$$
I(C)>I\left(C_{0}\right)
$$

holds for every admissible arc $C \neq C_{0}$ with elements $(a, b, y, z)$ in $\mathfrak{R}$. Since $l^{0}=1$ we have $J(C)=I(C)$. Consequently the hypotheses of Corollary .2 to Theorem 7.2 are satisfied and the inequality (10.1) holds for every admissible arc $C \neq C_{0}$ in a sufficiently small neighborhood $\mathfrak{F}$ of $C_{0}$ in $a y$-space.

We have just shown that if $C_{0}$ is a minimizing arc for the transformed problem, which is a problem of Mayer, then it is a minimizing arc for the original problem. The sufficiency conditions usually given are invariant under the transformation here used. It follows therefore that with the help of Theorem 7.2 the sufficiency theorems for the problem of Bolza can be obtained from those for the problem of Mayer.

11. Further results. In Theorem 7.1 it was shown that if $J(C)$ is close to $J\left(C_{0}\right)$ and $C^{\prime}, C_{1}$ are subarcs whose end values are close, then $H\left(C^{\prime}\right)$ is close to $H\left(C_{1}\right)$. The following theorem is concerned with conditions on $H(C)$ which imply that if $J(C)$ is close to $J\left(C_{0}\right)$, and $H\left(C^{\prime}\right)$ is close to $H\left(C_{1}\right)$, then the second end value of $C^{\prime}$ will be close to that of $C_{1}$ provided their initial end values are close.

Theorem 11.1. Suppose that the integrand $H(a, y, p)$ of $H(C)$ is E-dominated by $F$ near $C_{0}$ on $\mathfrak{D}$ and that $H(a, y, p)$ is positive along $C_{0}$. Then given a constant $\rho>0$ there exist positive constants $\epsilon, \eta$ and a neighborhood $\mathfrak{F}$ of $C_{0}$ in ay-space such that if $C$ is an admissible arc.in $\mathfrak{F}$ satisfying the condition

$$
J(C) \leqq J\left(C_{0}\right)+\eta
$$

and $C^{\prime}, C_{1}$ are subarcs of $C, C_{0}$ having the same initial points as $C, C_{0}$ and satisfying the condition

$$
\left|H\left(C^{\prime}\right)-H\left(C_{1}\right)\right|<\epsilon,
$$

then the end values of $C^{\prime}$ are in the p-neighborhood of those of $C_{1}$.

In order to prove this result let $b$ be a positive constant such that $L(p) \leqq b H(a, y, p)$ on $C_{0}$. Let $\rho$ be a positive constant and choose $\epsilon>0$ such that $4 b \epsilon<\rho$. Let $\mathfrak{F}, \delta, \eta$ be related to $\epsilon$ as described in Theorem 7.1. Diminish $\delta$ so that $2 \delta<\rho$. Diminish $\mathfrak{F}$ so that $\mathfrak{F}$ lies in the $\delta$-neighborhood of $C_{0}$ and so that if $C$ is an admissible arc in $\mathfrak{F}$ its end values lie in the $\delta$-neighborhood of those on $C_{0}$. Consider now arcs $C, C^{\prime}, C_{1}$ chosen as described in the theorem. Let $C_{2}$ be a subarc of $C_{0}$ whose initial point coincides with that of $C_{0}$ and whose final end point is in the $\delta$-neighborhood of the final end point of $C^{\prime}$. Then by Theorem 7.1 we have

$$
\left|H\left(C^{\prime}\right)-H\left(C_{2}\right)\right|<\epsilon .
$$

It follows that

$$
\left|H\left(C_{1}\right)-H\left(C_{2}\right)\right| \leqq\left|H\left(C_{1}\right)-H\left(C^{\prime}\right)\right|+\left|H\left(C^{\prime}\right)-H\left(C_{2}\right)\right|<2 \epsilon .
$$


Inasmuch as $L(p) \leqq b H(a, y, p)$ on $C_{0}$ we have

$$
\left|L\left(C_{1}\right)-L\left(C_{2}\right)\right| \leqq b\left|H\left(C_{1}\right)-H\left(C_{2}\right)\right|<2 b \epsilon<\rho / 2 .
$$

In view of this result and our choice of $C_{2}$ it follows that the end values of $C^{\prime}$, $C_{1}$ are in the $(\rho / 2)$-neighborhood of those on $C_{2}$. The end values of $C^{\prime}$ are accordingly in the $\rho$-neighborhood of those on $C_{1}$ as was to be proved.

As an application of Theorem 11.1 we have the following theorem.

THEOREM 11.2. Suppose each admissible arc $C$ has been given the representation

C:

$$
a, \quad y(t)
$$$$
(0 \leqq t \leqq 1)
$$

such that $|\dot{y}(t)|=L(C)$ for almost all values of $t$ on $0 \leqq t \leqq 1$ and let $C_{0}$ be defined by the set

$C_{0}$ :

$$
a_{0}, \quad y_{0}(t)
$$$$
(0 \leqq t \leqq 1) .
$$

If $L$ is $E$-dominated by $F$ near $C_{0}$ on $\mathfrak{D}$; then given a constant $\rho>0$ there is a constant $\eta>0$ and $a$ neighborhood $\mathfrak{F}$ of $C_{0}$ in ay-space such that the inequality

$$
\left|y(t)-y_{0}(t)\right|<\rho
$$

holds for every admissible arc $C$ in $\mathfrak{F}$ satisfying the condition (11.1).

For choose $\epsilon, \eta, \mathfrak{F}$ related to $\rho$ and $H(C)=L(C)$ as described in the last theorem. Diminish $\eta$ and $\mathfrak{F}$ so that they are related to $\epsilon$ and $H(C)=L(C)$ as described in Theorem 5.2. Consider now an admissible arc $C$ in $\mathfrak{F}$ satisfying the condition (11.1). By Theorem 5.2 we have

$$
\left|L(C)-L\left(C_{0}\right)\right|<\epsilon .
$$

Let $C^{\prime}, C_{1}$ be the subarcs of $C, C_{0}$ determined by the range $0 \leqq t \leqq t^{1}$ of the parameter $t$. Since $|\dot{y}(t)|=L(C)$ we have $L\left(C^{\prime}\right)=t^{1} L(C)$. Similarly $L\left(C_{1}\right)$ $=t^{1} L\left(C_{0}\right)$. We have accordingly

$$
\left|L\left(C^{\prime}\right)-L\left(C_{1}\right)\right|=t^{1}\left|L(C)-L\left(C_{0}\right)\right|<t^{1} \epsilon \leqq \epsilon,
$$

by (11.4). It follows from Theorem 11.1 that the end points of $C^{\prime}$ are in the $\rho$-neighborhood of those of $C_{1}$ and hence that the inequality (11.3) holds with $t=t^{1}$. This proves the theorem.

12. The function $K\left(C, C_{0}\right)$. It will be convenient at this time to study some of the properties of a function $K\left(C, C_{0}\right)$ that will be useful in the sufficiency theorem given in a forthcoming paper. The results here given are of interest in themselves apart from their applications to be made later.

In order to define $K\left(C, C_{0}\right)$ we shall suppose that each admissible arc $C$ has been given the representation

C:

$a, \quad y(t)$

$(0 \leqq t \leqq 1)$ 
such that for almost all values of $t$ on $0 \leqq t \leqq 1$ we have

$$
|\dot{y}(t)|=L(C) \text {. }
$$

The arc $C_{0}$, in particular, has the representation

$C_{0}$ :

$$
a_{0}, \quad y_{0}(t)
$$

such that

$$
\left|\dot{y}_{0}(t)\right|=L\left(C_{0}\right) \text {. }
$$

We define $K\left(C, C_{0}\right)$ by the formula

$$
\text { 3) } K\left(C, C_{0}\right)=\left|a-a_{0}\right|^{2}+\max _{0 \leqq t \leqq 1}\left|y(t)-y_{0}(t)\right|^{2}+\int_{0}^{1}\left|\dot{y}(t)-\dot{y}_{0}(t)\right|^{2} d t \text {. }
$$

Our principal result is given in the following theorem.

THEOREM 12.1. Given a constant $\epsilon>0$ there exist a neighborhood $\mathfrak{F}$ of $C_{0}$ in ay-space and a constant $\eta>0$ such that the inequality

$$
K\left(C, C_{0}\right)<\epsilon
$$

holds for every admissible arc $C$ in $\mathfrak{F}$ satisfying the condition

$$
J(C) \leqq J\left(C_{0}\right)+\eta \text {. }
$$

In order to prove this result we can suppose without loss of generality that the functions $p^{i}(a, y)$ described in $\$ 2$ have been chosen so that

$$
|p(a, y)|=L\left(C_{0}\right) \text {. }
$$

This result can be brought about by replacing $p^{i}(a, y)$ by $k(a, y) p^{i}(a, y)$, where $k=L\left(C_{0}\right) /|p|$, an operation that is permissible because of the homogeneity properties of admissible functions $H(a, y, p)$. To each arc $C$ there is accordingly associated a vector function

$$
p(t)=p[a, y(t) 1 \quad(0 \leqq t \leqq 1)
$$

of the parameter $t$ such that

$$
|p(t)|=L\left(C_{0}\right) .
$$

Because of the relations (12.6) and (12.2) the vector $p_{0}(t)$ associated with $C_{0}$ is identical with $\dot{y}_{0}(t)$. With the help of the law of the mean applied to the difference

$$
p(t)-p_{0}(t)=p[a, y(t)]-p\left[a_{0}, y_{0}(t)\right]
$$

it is seen that there is a constant $M$ such that the inequality

$$
\left|p(t)-p_{0}(t)\right| \leqq M\left[\left|a-a_{0}\right|+\left|y(t)-y_{0}(t)\right|\right]
$$


holds for every admissible arc $C$ in a neighborhood $\mathfrak{F}_{1}$ of $C_{0}$ in $a y$-space. Using this result and the relation

$$
\left|\dot{y}(t)-\dot{y}_{0}(t)\right| \leqq|\dot{y}(t)-p(t)|+\left|p(t)-p_{0}(t)\right|
$$

it follows readily that one can select a number $N$ so that

$$
K\left(C, C_{0}\right) \leqq N\left(K_{1}+K_{2}\right)
$$

where

$$
\begin{aligned}
& K_{1}=\left|a-a_{0}\right|^{2}+\max _{0 \leqq t \leqq 1}\left|y(t)-y_{0}(t)\right|^{2}, \\
& K_{2}=\int_{0}^{1}|\dot{y}(t)-p(t)|^{2} d t .
\end{aligned}
$$

We observe further that because of the identity

$$
|q-p|^{2}=[|q|-|p|]^{2}+2|p| E_{L}(p, q)
$$

and the relations (12.1) and (12.6) one has

$$
K_{2}=\left[L(C)-L\left(C_{0}\right)\right]^{2}+2 L\left(C_{0}\right) E_{L}^{*}(C) .
$$

It is clear from these relations between $K, K_{1}, K_{2}$ that the inequality (12.4) will hold if we show that $\mathfrak{F}$ and $\eta$ can be chosen so that given an arc $C$ in $\mathfrak{F}$ satisfying the condition (12.5) one has

$$
\begin{array}{r}
\left|a-a_{0}\right|^{2}<\epsilon / 4 N \\
\left|y(t)-y_{0}(t)\right|^{2}<\epsilon / 4 N \\
\left|L(C)-L\left(C_{0}\right)\right|^{2}<\epsilon / 4 N \\
* \\
2 L\left(C_{0}\right) E_{L}(C)<\epsilon / 4 N .
\end{array}
$$

The inequality (12.7) is obtained by a suitable choice of $\mathfrak{F}$. The condition (12.8) can be satisfied by virtue of Theorem 11.2 with $4 N \rho^{2}=\epsilon$. The condition (12.9) can be satisfied by virtue of Theorem 5.2 with $H(C)=L(C)$. In order to show that $\eta$ and $\mathfrak{F}$ can be diminished further so that (12.10) holds we first select $\eta$ so that

$$
16 N L\left(C_{0}\right) b \eta<\epsilon
$$

where $b$ is the constant appearing in (2.7). Let $\mathfrak{F}$ be related to $\eta$ as described in Theorem 4.2. Diminish $\mathfrak{F}$ further so that the end values of an admissible arc $C$ in $\mathfrak{F}$ are in the $\delta$-neighborhood of those of $C_{0}$ prescribed in Theorem 4.2. Then by the last statement of Theorem 4.2 and the relation (12.5) we have

$$
E_{F}^{*}(C)<2 \eta \text {. }
$$


It follows from (2.22) and (12.11) that

$$
8 N L\left(C_{0}\right) E_{L}^{*}(C) \leqq 8 N L\left(C_{0}\right) b E_{F}^{*}(C) \leqq 16 N L\left(C_{0}\right) b \eta<\epsilon .
$$

Thus (12.10) holds and the proof of Theorem 12.1 is complete.

THEOREM 12.2. Consider a sequence of admissible arcs $\left\{C_{q}\right\}$ converging to $C_{0}$ in the sense that given a neighborhood $\mathfrak{F}$ of $C_{0}$ in ay-space, there is an integer $q_{0}$ such that if $q>q_{0}$ then $C_{q}$ is in F. If

$$
\lim \sup J\left(C_{q}\right) \leqq J\left(C_{0}\right)
$$

then

$$
\lim _{q=\infty} K\left(C_{q}, C_{0}\right)=0, \quad \lim _{q=\infty} J\left(C_{q}\right)=J\left(C_{0}\right)
$$

and

$$
\lim _{q=\infty} H\left(C_{q}\right)=H\left(C_{0}\right)
$$

if the integrand $H(a, y, p)$ of $H(C)$ is $E$-dominated by $F(a, y, p)$ near $C_{0}$ on $\mathfrak{D}$.

This result follows readily from Theorems $12.1,4.2$, and 5.1.

The University of Chicago,

Chicago, Ill. 